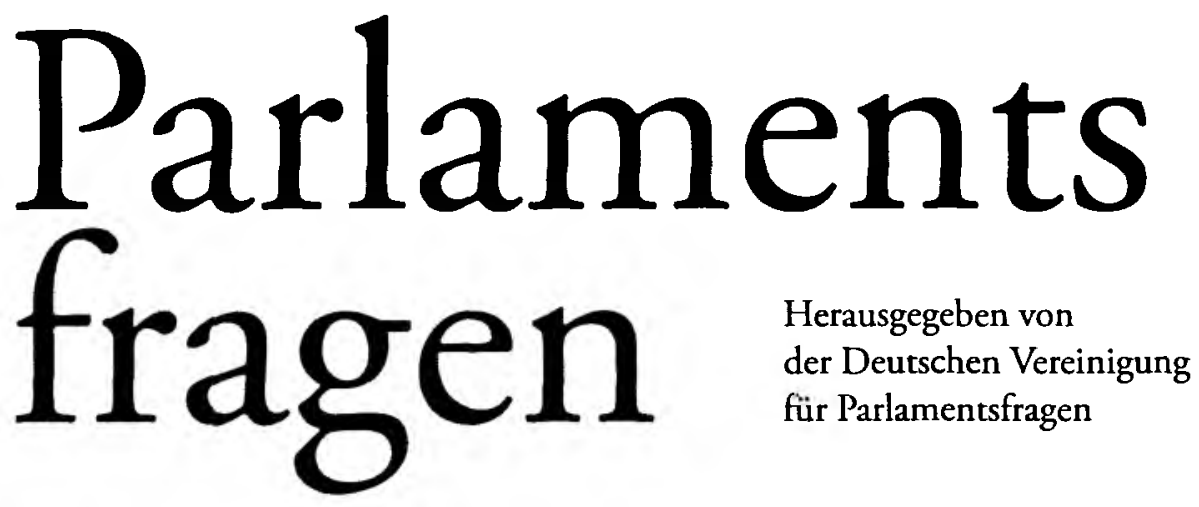

39. Jahrgang 2008 


\section{Editionsprogramm}

Normativ und empirisch demokratischer Politik verpflichtet, stehen Theorie und Wirklichkeit des Parlamentarismus im Zentrum des Redaktionsprogramms. Die Daten, Deskriptionen und Deutungen des Periodikums gelten insbesondere der Erforschung gegenwärtiger Legitimations- und Repräsentationsfragen. Die Zeitschrift für Parlamentsfragen (ZParl) wird zwar wissenschaftlich, aber nicht nur für Wissenschaftler gemacht; sie richtet sich an alle Studierenden und Vermittler einer schwierigen und stets entwicklungsbedürftigen Regierungsform.

\section{Editionsverfahren}

Die Redaktion der ZParl ist aus Politikwissenschaftlern und Juristen zusammengesetzt. Jedes eingesandte Manuskript durchläuft ein redaktionelles Begutachtungsverfahren, an dem alle Redaktionsmitglieder - Autoren wie Lesern gegenüber unmittelbar verantwortlich - teilnehmen und das zugleich für die Identität und Qualität dieser Zeitschrift bürgt.

\section{Impressum}

Herausgeber: Deutsche Vereinigung für Parlamentsfragen, Vorsizzender: Joachim Hörster, MdB, Geschäftsführer: Gunter Gabrysch, Plazz der Republik 1, 11011 Berlin, Telefon: 030/22 792658 , Fax: 030 / 22792655 , dvpari@t-online.de

Redaktionsanschrift: Prof. Dr. Suzanne S. Schüttemeyer (V.i.S.d.P.), Martin-Luther-Universität Halle-Wittenberg, Institut für Politikwissenschaft, 06099 Halle (Saale), Tel.: 0345 / 5524221 ] oder $0345 / 5524$ 267, Fax: $0345 / 552738$ 2, zparl@politik.uni-halle.de

Koordination für Osterreich: Prof. Dr. Heinz Fischer und Prof. Dr. Anton Pelinka; für die Wiederlande: Willem Bieedveld und Prof. Dr. M. P. C. M. van Schendelen; für die Schweiz: lic iur. Mariangela Wallimann-Bornatico.

Verlag: NOMOS Verlagsgesellschaft mbH \& Co. KG, Postfach 100310,76484 Baden-Baden, Tel.: 07221 / 21 04-0, Fax: 07221 / 21 04-43, nomos@nomos.de.

Internet: www.xparl.de

Nachdruck und Vervielfaltigung. Die Zeitschrift und alle in ihr enthaltenen einzelnen Beiträge sind urheberrechtlich geschüzz. Jede Verwernung außerhalb der engen Grenzen des Urheberrechtsgesetzes ist ohne Zustimmung des Verlages unzulässig. Das gilt insbesondere für Vervielfaltigungen, Übersetzungen, Mikroverfilmungen und die Einspeicherung und Verarbeitung in elektronischen Systemen.

Bezugshedingungen: Die Zeitschrift erscheint viermal im Jahr. Jahrespreis 46,- $€$, für Studenten und Referendare (unter Einsendung eines Studiennachweises) jährlich 31, $€$. Die Preise verstehen sich ind. MwSt. zzgl. Versandkosten. Kündigung jeweils drei Monate vor Kalenderjahresende.

Jährlich kann ein Sonderheft erscheinen, das nach Umfang berechnet und den Abonnenten des laufenden Jahrgangs mit einem Nachlass von $25 \%$ des jeweiligen Ladenpreises geliefert wird. Bei Nichtgefallen kann das Sonderheft innerhalb einer Frist von drei Wochen zurückgegeben werden.

Anzeigen: sales friendly Verlagsdienstleistungen, Bertina Roos, Siegburger Str. 123, 53229 Bonn, Telefon: 0228 / 97898-0, Fax: 02 28 / 97898-20, roosesales-friendly.de 


\title{
Parlamentsvergrößerung als Problemlösung für Überhangmandate, Pattsituationen und Mehrheitsklauseln ${ }^{*}$
}

\author{
Friedrich Pukelsheim und Sebastian Maier
}

In Wahlsystemen, die eine Personenwahl mit einer Verhältniswahl verbinden, können Überhangmandate entstehen. Außerdem können Pattsituationen auftreten, und die Umsetzung der absoluten Stimmenmehrheit in die absolute Sitzmehrheit im Parlament ist nicht ohne weiteres gesichert. Zur Lösung dieser Probleme sollen im Folgenden Strategien zur Vergrößerung des Parlaments vorgestellt werden. Anlass für diese Vorschläge sind Initiativen im Landtag von Nordrhein-Westfalen, das Landeswahlgesetz zu novellieren. Statt der bisherigen, doppelt ausgewerteten Einzelstimme soll ein an das Bundeswahlgesetz angelehntes System mit zwei Stimmen eingeführt werden. Zudem soll für die Verrechnung von Stimmen in Sizze die Quotenmethode mit Ausgleich nach größten Resten (Hare / Nie-

Überarbeitung der schriftlichen Stellungnahme für die öffentliche Anhörung des Hauptausschusses des Landtags Nordrhein-Westralen am 16. August 2007. Neben den Autoren, die Mathernatiker sind, wurden der Politikwissenschaftler Uwe Andersen, die Verfassungsrechtler Thorsen Koch und Bodo Pieroth, der Geschäftsführer des Vereins Mehr Demokratie Daniel Schily und das Vorstandsmitglied der LandesschülerInnenvertretung Nordrhein-Westfalen Horst Wenzel angehört. Weicere Informationen sind unter www.uni-augsburg.de/pukelsheim/2007Duesseldorf bereitgestellt. Die Autoren danken Martin Fehndrich (www.wahlrecht.de) und Benjamin Beckmann fiü wertvolle Kritik und Anregungen. 
meyer) durch die Divisormethode mit Standardrundung (Sainte-Laguë / Schepers) ersetzt werden. Auch wird diskutiert, das Wahlalter von 18 auf 16 Jahre zu senken. ${ }^{1}$

Als Beitrag zur Meinungsbildung der Fraktionen und des Parlaments wurde vom zuständigen Hauptausschuss ein Fragenkatalog für eine Sachverständigenanhörung erarbeitet. Von den neun Fragen werden hier die Fragen 4 und 5 zum Wahlsystem erörtert: „4. Ist die Umstellung auf das Divisorverfahren nach Sainte-Laguë ein richtiger Schritt?" und "5. Sind bei der Formulierung der Ausgleichsmandateregelung Probleme zu erwarten?" Als Mathematiker bejahen wir die beiden Fragen ohne Einschränkungen.

\section{Die Divisormethode mit Standardrundung (Sainte-Laguë / Schepers)}

Die Normierung der einzuführenden Divisormethode mit Standardrundung (Sainte-Laguë I Schepers) in $\$ 33$ der Drucksache 14/3978 ist überlagert von Elementen, die der zu ersetzenden Quotenmethode mit Ausgleich nach größten Resten (Hare / Niemeyer) und der lang gedienten Divisormethode mit Abrundung (D'Hondt / Hagenbach-Bischoff) angehören. Daher werden zunächst Verbesserungsvorschläge für die Absätze 2 bis 4 von $\$ 33$ gemacht, die die neue Methode in transparenterem Licht erscheinen lassen.

Bisher gaben die Wähler in Nordrhein-Westfalen eine Stimme ab, mit der sie den Bewerber einer Partei wählten. Die Stimme wurde einmal zu Gunsten des Wahlkreisbewerbers und ein zweites Mal zu Gunsten der Landesliste seiner Partei ausgewertet. Die Doppelauswertung von Einzelstimmen soll nun durch ein Zweistimmen-System wie bei Bundestagswahlen ersetzt werden. Die Bezeichnungen „Erststimme“ und "Zweitstimme" verführen dabei bekanntlich zum Irrglauben, die eine Stimme sei erstrangig und die andere zweitrangig. Daher sollten Begriffe gebildet werden, die dieser Fehlauffassung entgegenwirken. Dem folgt der Text in Drucksache 14/3978, der von "Wahlkreis-Stimme" (Erststimme) und "Landeslisten-Stimme" (Zweitstimme) spricht. ${ }^{2}$

In $\$ 33$ wird die eigentliche Verhältnisrechnung geregelt. Zunächst legt Absatz 2 fest, welche Landeslisten-Stimmen in die Rechnung eingehen. Wichtigster Hinderungsgrund ist die Fünfprozenthürde. Der Entwurf nimmt die Perspektive der Parteien ein: „Diese Parteien bleiben bei der Sitzverteilung unberücksichtigt." Unsere Formulierung gibt dagegen der Wählersicht den Vorrang und führt für die Wählerstimmen das Attribut der „Zuteilungsberechtigung " ein. ${ }^{3}$ Ein zweiter Grund, warum Stimmen nicht in die Verhältnisrechnung eingehen, ist der Doppelerfolgsausschluss. Wenn die Wahlkreis-Stimme einem Bewerber zum Sieg verhilft, dessen Sitz nicht dem Verhältnisausgleich unterworfen wird, so verliert die zugehörige Landeslisten-Stimme die Zuteilungsberechtigung. ${ }^{4}$ Folglich lautet unser Formulierungsvorschlag für $\$ 33$ Abs. 2:

1 Hinzu kommen weitere Aktualisierungen und Anpassungen, siehe die Gesetzentwürfe der Fraktion Bündnis 90/Die Grünen (Drucksache 14/719) und der Landesregierung (Drucksache 14/3978).

2 In Hessen, Rheinland-Pfalz und Thūringen wird das Begriffspaar Wahlkreisstimme und Landesstimme benutzt, in Sachsen Direktstimme und Listenstimme und in Sachsen-Anhalt Personenstimme und Parteienstimme.

3 Friedrich Pukelsheim, Erfolgswertgleichheit der Wählerstimmen zwischen Anspruch und Wirklichkeit, in: Die öffentliche Verwaltung, 57. Jg. (2004), S. 405-413, S. 406.

4 Der Doppelerfolg bei Wahlkreissiegern, deren Partei eine Landesliste aufstelle, dann aber an der Fünfprozenthürde scheitert, wird in Drucksache $14 / 719$ bedacht und in $14 / 3978$ vergessen. 
„Der Landeswahlausschuss zählt die gültigen Landeslisten-Stimmen für jede Landesliste zusammen und stellt ihre Zuteilungsberechtigung fest. Eine Landeslisten-Stimme ist zuteilungsberechtigt, wenn sie für eine Partei abgegeben wurde, die mindestens fünf Prozent der gültigen Landeslisten-Stimmen erhalten hat, und wenn mit der zugehörigen Wahlkreis-Stimme nicht ein Wahlkreissieger gewählt wurde, der Einzelbewerber ist oder Bewerber einer Wählergruppe oder Partei, die keine Landesliste aufgestellt oder deren Landesliste weniger als fünf Prozent der gültigen Landeslisten-Stimmen erhalten hat. Die zuteilungsberechtigten Landeslisten-Stimmen werden der Verhältnisrechnung zugrunde gelegt.“

Absatz 3 legt die Zahl der Sitze fest, die in die Verhältnisrechnung eingehen. Im Wesentlichen ist das die in $\$ 14$ Abs. 2 genannte Ausgangszahl von 181 Mandaten. Davon abzuziehen sind solche Sitze, die auf Grund der Persönlichkeitswahlkomponente an der Verhältnisrechnung vorbeilaufen ${ }^{5}$ :

„Die Ausgangssitzzahl für die Verhältnisrechnung ist die Landtagsgröße aus $\$ 14$ Abs. 2, vermindert um die Zahl der Wahlkreissitze von Einzelbewerbern oder von Bewerbern von Wählergruppen oder Parteien, die keine Landesliste aufgestellt oder deren Landesliste weniger als fünf Prozent der gültigen Landeslisten-Stimmen erhalten haben."

Die Entwurfsabsätze 4 und 5 widmen sich der Divisormethode mit Standardrundung (Sainte-Laguë/ Schepers). Demgegenüber erscheint es übersichtlicher, die einzelnen Schritte in vier Absätze zu glíedern. Entsprechend beschreibt unser Absatz 4 die eigentliche Divisorrechnung, die dem Motto "Teile und runde!" 6 folgt:

„Zur Berechnung der Sitzzuteilung werden die Landeslisten-Stimmen der Parteien durch einen gemeinsamen Divisor geteilt und die Ergebnisse zur nächsten ganzen Zahl gerundet; der Divisor wird so bestimmt, dass die Ausgangssitzzahl aus Absatz 3 ausgeschöpft wird.“

Die Rechnung gemäß Absatz 4 ist denkbar einfach, wie die Anwendung auf das Wahlergebnis für die laufende 14. Wahlperiode (WP) zeigt. Dabei behandeln wir die vormaligen Einzelstimmen wie zukünftige Landeslisten-Stimmen. Jede Zahl zwischen 42 783,1 und 43 233,9 kann als Divisor dienen; in diesem Bereich ist 43000 ein ansprechender Wert. Er besagt, dass auf je 43000 Wählerstimmen rund ein Landtagssitz entfällt.

\begin{tabular}{|lrrrrrr|}
\hline & CDU & \multicolumn{1}{c}{ SPD } & Grüne & \multicolumn{1}{c|}{ FDP } & Summe & Divisor \\
\hline Stimmen & 3696506 & 3058988 & 509293 & 508266 & 7773053 & \\
Teilungsergebnis & 85,97 & 71,14 & 11,84 & 11,82 & & 43000 \\
Sitze & 86 & 71 & 12 & 12 & 181 & \\
\hline
\end{tabular}

Unser Formulierungsvorschlag für Absatz 4 schweigt darüber, wie ein passender Divisor gefunden wird. Drei Rechenwege sind bekannt. Beim ersten werden die Stimmenzahlen durch die Teiler $0,5,1,5,2,5$ usw. dividiert und die Sizze fortlaufend nach absteigenden "Höchstzahlen" vergeben. (Diese Rechenart ist von der Divisormethode mit Abrundung (D'Hondt / Hagenbach-Bischoff) her vertraut, nur dass dort die Teiler 1, 2, 3 usw. benutzt

5 Dieser Ausnahmefall ist allerdings bei den vergangenen vierzehn nordrhein-westfälischen Landtagswahlen nicht aufgetreten.

6 Das freie Computerprogramm Bazi (www.uni-augsburg.de/bazi) kann benutzt werden, einen geeigneten Divisor zu bestimmen. 
werden.) Beim zweiten Rechenweg werden statt der Höchstzahlen deren Kehrwerte betrachtet und die Sizze fortlaufend nach diesen aufsteigenden „Rangmaßzahlen “ zugeteilt. ${ }^{7}$ Der dritte Rechenweg ist der schnellste. Startdivisor ist die durchschnittliche Stimmenzahl pro Sitz, also der Quotient aus Gesamtstimmenzahl und Landtagsgröße. Die Stimmenzahlen der Parteien werden durch den Startdivisor geteilt und dann standardmäßig - das heißt zur nächstgelegenen ganzen Zahl - auf- beziehungsweise abgerundet. Sollte der Rest genau gleich einem Halben sein, liegen die beiden benachbarten ganzen Zahlen gleich nah und man darf auf- oder abrunden.

Schöpfen die so berechneten Sirzzahlen die Landtagsgröße aus, stellen sie das Endergebnis dar. Dies wird auf grob zwei Drittel aller Anwendungsfälle zutreffen. Beim restlichen Drittel wird die Landtagsgröße um einen Sitz verfehlt. ${ }^{8}$ Ist es einer zu wenig, wird der Divisor so verkleinert, dass ein weiteres Mandat vergeben wird und die Zielsitzzahl ausgeschöpft ist. Wurde zunächst ein Sitz zu viel verteilt, wird der Divisor entsprechend vergröBert. Wie gerechnet wird, um das Zuteilungsergebnis zu bestimmen, sollte nicht gesetzlich normiert, sondern dem Vollzug überlassen werden. ${ }^{9}$

\section{Ausgleichsmandateregelung}

Da bei der nordrhein-westfälischen Landtagswahl 2005 die CDU mehr Wahlkreissieger stellte (89), als ihr die Ausgangsrechnung an Sitzen zuteilte (86, siehe oben), kommt die Ausgleichsmandateregelung zur Anwendung. Wir schlagen vor, den Landtag zu vergrößern, bis alle Wahlkreissieger im Verhältnisausgleich Platz finden. Dann wäre 2005 auf 41700 Stimmen rund ein Sitz entfallen, und dem Landtag hätten 186 Abgeordnete angehört. ${ }^{10}$ Tatsächlich ist der amtierende Landtag größer und umfasst 187 Mitglieder.

\begin{tabular}{|lrrrrrr|}
\hline 14. WP (128 Wkr) & \multicolumn{1}{c}{ CDU } & \multicolumn{1}{c}{ SPD } & Grüne & FDP & Summe & Divisor \\
\hline Stimmen & 3696506 & 3058988 & 509293 & 508266 & 7773053 & \\
Sitze + Überhang & $86+3$ & 71 & 12 & 12 & $181+3$ & 43000 \\
& $\vdots$ & $\vdots$ & $\vdots$ & $\vdots$ & $\vdots$ & \\
Sizze + Überhang & $88+1$ & 73 & 12 & 12 & $185+1$ & 42000 \\
Sitze & 89 & 73 & 12 & 12 & 186 & 41700 \\
Sizze (22. Mai 2005) & 89 & 74 & 12 & 12 & 187 & 41500 \\
\hline
\end{tabular}

7 Peter Schindler, Datenhandbuch zur Geschichte des Deutschen Bundestages 1949 bis 1999, Baden-Baden 1999, Band II, S. 2085.

8 Bei mehr als vier Parteien kann das Zwischenergebnis auch um mehr als einen Sirz danebenliegen.

9 Formulierungsvorschläge für Vollzugshinweise, die in die Landeswahlordnung Eingang finden könnten, stehen unter www.uni-augsburg.de/bazi/DivStd.pdf bereit.

10 Die CDU bekommt mindestens 89 Size, wenn der Divisor kleiner wird als $3696506 / 88,5=$ 41768,4 . 
Im nordrhein-westfälischen Landeswahlgesetz wurden bisher drei Ausgleichsmandateregelungen normiert; unser Vorschlag für $\$ 33$ Abs. 5 kehrt zur ersten derartigen Regelung zurück $^{11}$ :

"Bleibr die Sitzzahl einer Partei unter der Zahl ihrer Wahlkreissieger, wird die Landtagsgröße erhöht, bis die erneute Sitzzuteilung für jede Partei eine Sitzzahl ergibt, die die Zahl ihrer Wahlkreissieger erreicht oder übertrifft."

Der Schein, dass der Landtag mit dieser Erhöhungsstrategie „aus allen Nähten platzen“ könnte, trügt. Unser Vorschlag hätte nicht nur 2005, sondern in allen Wahlperioden seit 1985 weniger Sitze für den Verhältnisausgleich benötigt als die Regelungen in $\$ 33$ Abs. 4 des geltenden Gesetzes und in $\$ 33$ Abs. 5 der Drucksache 14/3978. Gesetz und Entwurf bestehen auf ungeraden Landtagsgrößen; selbst dann führen sie 1995 und $1990 \mathrm{zu}$ einem Überausgleich. Es ist kein Grund ersichtlich, ungeraden Landtagsgrößen den Vorzug zu geben, denn schließlich ist die knappste Mehrheit bei gerader Landtagsgröße (wie 91:89 bei 180) doppelt so komfortabel wie bei ungerader (wie $91: 90$ bei 181). Sollten wie bisher nur ungerade Sitzzahlen gewünscht werden, müsste im obigen Vorschlag die Landtagsgröße in Zweierschritten erhöht werden. Die größte Schwäche der geltenden Ausgleichsmandateregelung geht aber in die Gegenrichtung, da sie bei unglücklicher Zahlenlage gar nichts verändert und insbesondere auch keine Ausgleichsmandate generiert. ${ }^{12}$

\section{Pattaufösung}

Patts sind zum Beispiel unvermeidlich, wenn aus der Wahl mehrere Parteien mit genau denselben Stimmenzahlen hervorgehen. Bei Landtagswahlen sind solche Gleichstände allerdings noch nie aufgetreten, so dass es keine empirischen Beispiele gibt. Keine Zuteilungsmethode kann ohne Zusatzregelungen alle Pattsituationen eindeutig auflösen. Um dem Problem aus dem Weg zu gehen, empfehlen wir auch hier eine Erhöhungsstrategie ${ }^{13}$ :

„\$33 Abs. 6 Genügen mehrere Sitzzuteilungen dieser Rechenvorschrift, wird die Landtagsgröße erhöht, bis die erneute Sitzzuteilung eindeutig wird."

Die Strategie, bei unbefriedigendem Zuteilungsergebnis den Landtag zu vergrößern, strahlt in der Gesamtschau eine problemangemessene Gelassenheit aus. Eine einzelne, feste Landtagsgröße bietet zu wenig Anpassungsgrade, um alle denkbaren Wahlergebnisse hinsichtlich der vielfältigen Anforderungen makellos abzubilden. Es steigert den inneren Zusammenhalt des Wahlsystems, die Erhöhungsstrategie gleichermaßen auf Ausgleichsmandateregelung und Pattaufösung anzuwenden. Da außer der Landtagsgröße alle anderen Komponenten

11 Vgl. Benjamin Beckmann, Das Landtagswahlsystem in Nordrhein-Westfalen, Diplomarbeit am Lehrstuhl Prof. Dr. Götz Trenkler, Fachbereich Statistik der Universität Dortmund, Dortmund 2006, S. 99 (www.wahlrecht.de/doku/download/2006-beckmann-landtagswahlrecht-nordrheinwestfalen.htm).

12 Beispiele für einen Unterausgleich geben www.wahlrecht.de/ueberhang/regelungsluecke.html und Benjamin Beckmann, a.a.O. (Fn. 11), S. 103. Unser Vorschlag vermeidet die Diskrepanz im geltenden Gesetz, dass Normierungsanspruch und Vollzugsvorschrift stark auseinanderklaffen.

13 Bei einem Gleichstand zwischen zwei Parteien reicht ein Zusatzsitz, bei drei Parteien zwei usw. Die Zahl der Zusatzsitze wird also immer um Eins hinter der Zahl der Gleichstandsparteien ztrückbleiben. 
des Zuteilungsverfahrens gleich bleiben, stehen dieser Strategie unseres Erachtens keine verfassungsrechtlichen Einwände entgegen.

Die Divisormethode mit Standardrundung (Sainte-Laguë / Schepers) liefert speziell für die vergangenen nordrhein-westfälischen Landtagswahlen dieselben Zuteilungsergebnisse wie die zu ersetzende Quotenmethode mit Ausgleich nach größten Resten (Hare / Niemeyer). Generell aber krankt letztere bei einer Erhöhung der Landtagsgröße an unlogischen Rücksprüngen, was sie für die von uns favorisierte Erhöhungsstrategie ungeeignet macht. ${ }^{14}$ Mehr Tradition hat eine alternative Pattauflösung, die unter den gleichberechtigten Zuteilungen einen Losentscheid herbeiführt und somit ohne Zusatzsitze auskommt. Dementsprechend lautet unser Alternativvorschlag:

n\$33 Abs. 6A Genügen mehrere Sitzzuteilungen dieser Rechenvorschrift, entscheidet unter diesen das vom Landeswahlleiter zu ziehende Los. ${ }^{.}$

\section{Mehrheitsklausel}

Die Mehrheitsklausel garantiert, dass einer Partei mit einer absoluten Mehrheit zuteilungsberechtigter Landeslisten-Stimmen in jedem Fall auch eine absolute Sitzmehrheit im Landtag zugewiesen wird. Das wird von allen vernünftigen Zuteilungsmethoden in den meisten Fällen automatisch geleistet, in seltenen Sonderfällen aber nicht. Mehrheitsklauseln sind dazu da, auch diese zu regeln. So sollte $\$ 33$ Abs. 7 wie folgt abgefasst werden ${ }^{15}$ :

„Erhält eine Partei mit mehr als der Hälfte der zuteilungsberechtigten Landeslisten-Stimmen nichr mehr als die Hälfte der Landtagssitze zugeteilt, werden ihr so viele weitere Sizze zugewiesen, bis sie über mehr als die Hälfte der Landtagssizze verfügt."

Ein Beispiel bietet die Wahl zum Beirat Gröpelingen im Gebiet der Stadt Bremen am 25. Mai 2003. ${ }^{16}$ Dort wurden der SPD trotz absoluter Stimmenmehrheit nur neun von 19 Sitzen zugeteilt. Unsere Regelung schafft zwei Zusatzsitze, um der Partei mit elf von 21 Sitzen die Mehrheit zu sichern. Für die Beiratsgröße 21 führt die Divisormethode mit Standardrundung (Sainte-Laguë / Schepers) zu demselben Zuteilungsergebnis, so dass trotz Mehrheitsklausel die Erfolgswertgleichheit keinen Schaden nimmt. Es gibt aber Fälle, in denen obiger Vorschlag von der erneuten Zuteilung bei erhöhter Landtagsgröße abweicht. ${ }^{17}$

Die Mehrheitsklausel in $\$ 6$ Abs. 3 Bundeswahlgesetz war auf die dort benutzte Quotenmethode mit Ausgleich nach größten Resten (Hare / Niemeyer) zugeschnitten. Zwar war

14 Peter Schindler, a.a.O. (Fn. 7), S. 2084.

15 Eine mit unserem Vorschlag übereinstimmende Mehrheitsklausel findet sich in Art. 42 Abs. 5 des Bayerischen Landeswahlgesetzes.

16 Siehe Beispiel 3 in www.uni-augsburg.de/bazi/DivStd.pdf.

17 In der schriftlichen Stellungnahme zur Anhörung sind wir der Erhöhungsstrategie für Ausgleichsmandateregelung und Pattauflösung gefolgt und haben gefordert, die Landtagsgröße zu erhöhen, bis die erneute Sitzzuteilung der Mehrheitspartei eine absolute Sitzmehrheit zuweist. Diese Formulierung versagt aber in denkbaren Extremfälen; wenn etwa drei Einzelbewerber Erfolg härten (was bisher noch nie der Fall war), dann müssten für eine absolute Mehrheit von den verbleibenden, der Verhältnisrechnung unterworfenen Sitzen ein Vorsprung von vier Sizzen herauskommen, was auch durch eine Vergrößerung der Sitzzahl nicht erreicht werden kann. Hilfsweise könnte $\$ 33$ Abs. 7 dahingehend modifiziert werden, dass nur mehr als die Hälfte der dem Verhältnisausgleich unterworfenen Sizze garantiert werden. 
sie vom Niedersächsischen Staatsgerichtshof geprüft und für mit den verfassungsrechtlichen Wahlgrundsätzen vereinbar befunden worden ${ }^{18}$; sie hatte aber den Nachteil, dass sie für keine der Divisormethoden tauglich ist. ${ }^{19}$

Das geltende nordrhein-westfälische Landeswahlgesetz und der Entwurf 14/3978 enthalten keine Mehrheitsklausel, bestehen aber - wie schon erwähnt - auf ungeraden Landtagsgrößen. Hier darf ein Zusammenhang vermutet werden, denn die lang gediente Divisormethode mit Abrundung (D'Hondt / Hagenbach-Bischoff) hat die Eigenschaft, dass sie bei ungerader Landtagsgröße eine absolute Stimmenmehrheit garantiert in eine absolute Sitzmehrheit abbildet und somit eine Mehrheitsklausel überflüssig macht. Allerdings ist diese Methode nie im nordrhein-westfälischen Landeswahlgesetz verwendet worden.

Auch für die Mehrheitsklausel gibt es eine alternative Regelungsmöglichkeit, um die vorgegebene Landtagsgröße zu wahren. Die Alternative behandelt die Mehrheitspartei getrennt von den Minderheitsparteien, um die Beziehung Mehrheit-Minderheit nicht anzutasten. Vorbild dafür ist die „itio in partes“ als Verfahrenprozedur aus dem Westfälischen Frieden von Münster und Osnabrück $1648 .{ }^{20}$ Daraus ergib sich für $\$ 33$ Abs. 7 folgender Alternativvorschlag:

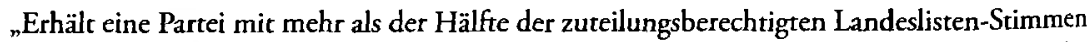
nicht mehr als die Hälfte der Landtagssitze zugeteilt, wird ihr statt dessen die kleinstmögliche absolure Sizzmehrheit zugewiesen; die übrigen Sitze werden den übrigen Parteien gemäß der Berechnungsvorschrift aus Absatz 4 neu zugeteilt."

\section{Verträglichkeit mit den Wahlgrundsätzen der Landesverfassung}

Art. 31 Abs. 1 der nordrhein-westfälischen Landesverfassung besagt: „Die Abgeordneten werden in allgemeiner, gleicher, unmittelbarer, geheimer und freier Wahl gewählt." Für die Beurteilung von Sitzzuteilungsmethoden ist der Grundsatz der gleichen Wahl ausschlaggebend. Das Bundesverfassungsgericht bezieht den Gleichheitsgrundsatz vorrangig auf die Wähler und präzisiert die abstrakte Wahlgleichheit zur konkreten „Erfolgswertgleichheit der Wählerstimmen“: „alle Wähler sollen mir der Stimme, die sie abgeben, den gleichen Einfluss auf das Wahlergebnis haben. " ${ }^{21}$ Weder das Bundesverfassungsgericht noch die Landesverfassungsgerichtshöfe engen ihre Auslegungen so weit ein, dass am Schluss nur eine einzige Zuteilungsmethode übrig bleibt. Bei der Ausgestaltung des Wahlsystems hat der Gesetzgeber eine erhebliche Gestaltungsfreiheit.

18 Nds.StGHE 1, 1978, S. 335 - 372 (www.wahlrecht.de/wahlpruefung/19770920.htm).

19 Das Sitzzuteilungsverfahren im Bundeswahlgeserz ist mit Änderungsgeserz vom 17. März 2008 auf die Divisormethode mit Standardrundung (Sainte-Laguë / Schepers) umgestellt worden. Die damit einhergehende Anpassung der Mehrheitsklausel ist allerdings nur unzureichend gegiüdkt, siehe Abschnitt $\mathrm{V}$ in Friedrich Pukelsheim, Bundeswahlgesez - Nächste Etappe, in: DVBI 14/2008.

20 Friedrich Pukelsheim / Sebastian Maier, Eine schonende Mehrheitsklausel für die Zureilung ron Ausschusssitzen, in: ZParl, 36. Jg. (2005), H. 4, S. $763-772$.

21 BVerfGE 1, 1952, S. 208 - 263, S. 246. 


\subsection{Erfolgswertgleichheit der Wählerstimmen}

Mit dem Wechsel zur Divisormethode mit Standardrundung (Sainte-Laguë / Schepers) entscheidet sich der nordrhein-westfälische Landtag für eine Methode, die dem Ziel der Erfolgswertgleichheit der Wählerstimmen so nahe kommt, wie dies mathematisch überhaupt nur möglich ist.

Der Erfolgswert einer Wählerstimme kann wie folgt als Zahl gefasst werden: Bei der Landtagswahl 2005 wurden 3696506 Stimmen für die CDU abgegeben, sie führten zu einem Erfolg von 89 Sitzen. Wenn alle Stimmen am Erfolg in gleicher Weise teilhaben, entfält auf eine einzelne Stimme der Erfolgsbruchteil $\frac{89}{3696506}$, was der Quotient aus Sitzzahl und Stimmenzahl ist. Hier fehlt aber noch der Bezug auf die Landtagsgröße und die Gesamtstimmenzahl, denn ob die 89 Sitze einen großen oder kleinen Erfolg bedeuten, wird erst im Licht der Landtagsgröße 187 sichtbar. Ebenso ist das Gewicht von 3696506 Wählerstimmen nur zu ermessen, wenn man die Gesamtstimmenzahl 7773053 mit bedenkt. Als „Erfolgswert einer Wählerstimme“ gilt daher der Quotient aus den Anteilen an Sitzen und Stimmen. So ergibt sich der Erfolgswert einer CDU-Wählerstimme zu 100,08 Prozent, einer SPD-Wählerstimme zu 100,56 Prozent, einer Grünen-Wählerstimme zu 97,94 Prozent und einer FDP-Wählerstimme zu 98,14 Prozent. ${ }^{22}$

Die theoretische Forderung nach der exakten Gleichheit dieser Erfolgswerte zielt auf Unmögliches, denn es gibt nur ganze Abgeordnete und keine Bruchteile von ihnen. Die Praxis fragt deshalb bescheidener: Sind diese Erfolgswerte so wenig ungleich wie möglich? Die Antwort hängt davon ab, wie man die Ungleichheiten bewertet. Zwei Zugänge seien hier dargestell $\mathbf{t}^{23}$ :

Für einen Vergleich von Erfolgswerten braucht es mindestens zwei Beteiligte. So unterscheiden sich die Erfolgswerte eines SPD-Wählers und eines Grünen-Wählers um 2,62 Prozentpunkte $(100,56$ - 97,94). Man könnte versuchen, durch einen Sitztransfer von der SPD zu den Grünen den Unterschied zu verkleinern. Der Versuch scheitert: Mit dann 106,10 - 99,20 = 6,90 Prozentpunkten wird der Erfolgswertunterschied mehr als doppelt so groß. ${ }^{24}$ Allgemeiner können die Wähler von je zwei Parteien versuchen, durch einen Sitztransfer ihren Erfolgswertunterschied zu verkleinern: CDU und SPD, CDU und Grüne, CDU und FDP, SPD und Grüne (siehe oben), SPD und FDP, schließlich Grüne und FDP. Alle diese Versuche sind ebenfalls zum Scheitern verurteilt. Die Divisormethode mit Standardrundung (Sainte-Lague / Schepers) ergibt immer eine Zuteilung, bei der jeglicher Sitztransfer die paarweisen Erfolgswertunterschiede nur größer und nie kleiner macht. In diesern ersten Sinn macht die Methode die Erfolgswerte zwischen je zwei Wählerstimmen so wenig ungleich wie möglich. ${ }^{25}$

22 CDU-Wählerstimme: $\frac{89 / 187}{3696506 / 7773053}=1,0008$; SPD-Wählerstimme: $\frac{74 / 187}{3058988 / 7773053}=1,0056$; Grünen-Wählerstimme: $\frac{12 / 187}{509293 / 7773053}=0,9794$; FDP-Wählerstimme: $\frac{12 / 187}{508266 / 7773053}=0,9814$.

23 Friedrich Pukelsheim, Mandatszuteilungen bei Verhälıniswahlen: Erfolgswertgleichheit der Wählerstimmen, in: Allgemeines Statistisches Archiv, 84. Jg. (2000), S. 447 - 459.

24 Grünen-Wăhlerstimme: $\frac{13 / 187}{509293 / 7773053}=1,0610$; SPD-Wählerstimme: $\frac{73 / 187}{3058988 / 7773053}=0,9920$

25 Vgl. Ladislaus von Bortkiewicz, Ergebnisse verschiedener Verteilungssysteme bei der Verhältniswahl, in: Annalen für soziale Politik und Gesetzgebung, 6. Jg. (1919), S. 592 - 613, S. 608. 
Beim zweiten Zugang wird eine einzige Kennzahl erzeugt, die ein Gesamtmaß für die Ungleichheit darstellt, die dem Zuteilungsergebnis innewohnt. Die Abweichung von einem vollen, hundertprozentigen Erfolg beträgt für eine CDU-Wählerstimme 100,08 - 100 = 0,08 , für eine SPD-Wählerstimme $100,56-100=0,56$, für eine Grünen-Wählerstimme $97,94-100=-2,06$ und für eine FDP-Wählerstimme 98,14 $-100=-1,86$. Um die gesamte Wählerschaft zu erfassen und zugleich größere Abweichungen stärker zu gewichten als kleinere und die Abweichungsrichtung zu neutralisieren, betrachtet man die Summe der Abweichungsquadrate:

$3696506 \cdot(0,08)^{2}+3058988 \cdot(0,56)^{2}+509293 \cdot(-2,06)^{2}+508266 \cdot(-1,86)^{2}=4902589,1036$

Es gibt keine Sitzzuteilungsmethode, bei der diese Kennzahl geringer ausfält als bei der Divisormethode mit Standardrundung (Sainte-Laguë/Schepers). Auch in diesem zweiren Sinn ist es gerade diese Methode, die die wenigste Ungleichheit mit sich bringt. ${ }^{26}$

Die Tatsache, dass die Divisormethode mit Standardrundung (Sainte-Laguë/Schepers) so hervorragend mit dem Grundsatz der Erfolgswertgleichheit der Wählerstimmen harmoniert, macht sie allen konkurrierenden Zuteilungsverfahren überlegen. Dass sie dabei den Vorstellungen der Verfassungsgerichtsbarkeit entgegenkommt, festigt das Zusammenwirken von Legislative und Jurisdiktion. Noch wichtiger erscheint, dass die Methode den Gleichheitsanspruch des demokratischen Souveräns in bestmöglicher Weise verwirklicht. Damit wird auch die Legitimationskraft für die Mandatsträger gestärkt. Alle Verantwortlichen in Politik und Gesellschaft können parteiübergreifend mit den besten Argumenten für dieses System bei den Wählern werben.

\subsection{Idealansprüche der Parteien}

In der modernen Parteiendemokratie muss eine Sitzzureilungsmethode neben aller demokratischen Verankerung auch die Parteien als staatstragende Institutionen, als die sie sich verstehen, mit erkennbarem Gleichheitsgehalt behandeln. Auch hier bewährt sich die neue Methode bestens. Die Idealansprüche, die die Parteien bei der Landtagswahl 2005 reklamieren konnten, betrugen für die CDU 88,93 Sitzbruchteile, für die SPD 73,59, für die Grünen 12,25 und für die FDP 12,23. ${ }^{27}$ Bei der Divisormethode mit Standardrundung (Sainte-Laguë / Schepers) erweisen sich die Unterschiede zwischen realen Sitzzahlen und idealen Ansprüchen als so gering, dass kein Sitztransfer zwischen zwei Parteien beide Betroffenen gleichzeitig besser stellen kann. Folgende Unterschiede ergeben sich: für die CDU $89-88,93=0,07$, für die SPD $74-73,59=0,41$, für die Grünen $12,25-12=0,25$, für die FDP $12,23-12=0,23$. Wenn durch einen Sitztransfer zwischen zwei Parteien die Unterschiede für beide kleiner würden, müsste die Zuteilung als angreifbar und instabil gelten.

$26 \mathrm{Vgl}$. André Sainte-Laguë, La représentation proportionnelle et la méthode des moindres carrés, in: Annales scientifiques de l'École normale supérieure, Troisiéme série, 27. Jg. (1910), S. $529-\$ 42$, S. 532.

27 CDU-Idealanspruch: $\frac{3696506}{7773053} \cdot 187=88,93$; SPD-Idealanspruch: $\frac{3058988}{7773053} \cdot 187=73,59$; Grünen-Idealanspruch: $\frac{509293}{7773053} \cdot 187=12,25$; FDP-Idealanspruch: $\frac{508266}{7773053} \cdot 187=12,23$. 
Die Sitzzuteilungen der Divisormethode mit Standardrundung (Sainte-Laguë / Schepers) sind dagegen gefeit. 28

Die mächtigste Stabilitätseigenschaft ist die der „Kohärenz “, des Zusammenhangs zwischen dem Ganzen und seinen Teilen. Wenn zwei Parteien um einen Sitz zu streiten beginnen, ist aus der ganzen Sitzzuteilung nur gerade jenes Teilergebnis berührt, das die beiden Kontrahenten betrifft. Die Divisormethode mit Standardrundung (Sainte-Laguë/Schepers) ist die einzige Sitzzuteilungsmethode, bei der keine von zwei beliebigen Streitparteien einen rechnerischen Anlass findet, der anderen einen Sitz wegzunehmen. Immer bekommen beide gerade so viele Sitze, wie ihre gerundeten Idealansprüche ausmachen, würden sie das umstrittene gemeinsame Sitzkontingent neu unter sich aufteilen. ${ }^{29}$

\subsection{Fünfprozenthürde}

Der Verfassungsgerichtshof für das Land Nordrhein-Westfalen hat 1999 über die Fünfprozenthürde im Kommunalwahlgesetz geurteilt. Der Grundsatz der gleichen Wahl sei wegen des Zusammenhangs mit dem egalitären demokratischen Prinzip im Sinne einer strengen und formalen Gleichheit zu verstehen. Differenzierungen würden stets eines zwingenden Grundes bedürfen. Bei einer Senkung des aktiven Wahlrechts auf 16 Jahre erschwere die unverminderte Beibehaltung der Fünfprozenthürde der neu hinzutretenden Wählergruppe, eine ihren besonderen Interessen gemäße Vertretung in den Landtag zu wählen. Dies böte Anlass, die weitere Erforderlichkeit der Fünfprozenthürde zu überdenken.$^{30}$ Die nun dem Landtag vorliegenden Gesezzesentwürfe (Drucksachen 14/719 und 14/3978) lassen nicht erkennen, dass der Gesetzgeber solche Überlegungen angestellt hat. Was die Effizienz der Arbeit des Parlaments betrifft, gibt seine Geschäftsordnung Hinweise. ${ }^{31} \mathrm{Gemäß} \$ \$ 65$ und 85 bedarf es mindestens sieben Abgeordneter, um Gesetzentwürfe oder Große Anfragen einzubringen. Die Gewährung wesentlicher Rechte an eine Minderheit von sieben Parlamentariern (3,7 Prozent) belegt, dass die Geschäftsordnung solche Minderheiten duldet. Warum das Landeswahlgesetz mit der Fünfprozenthürde die Messlatte höher legt, bleibt dahingestellt.

Ist der Gesetzgeber besorgt, dass die Parteien dem Anspruch an politische Institutionen gerecht werden und beim Wahlvolk so viel Zuspruch finden, dass sie sowohl im Parlament als auch im Land wirksame Aktivitäten erwarten lassen, dann wäre eine Dreiprozenthürde

$28 \mathrm{Vgl}$. Michel Balinski / Peyton Young, Fair Representation. Meeting the Ideal of One Man, One Vore, 2. Auflage, Washington DC 2001, S. 132. Die Nähe zu den Idealansprüchen kommt auch dadurch zum Ausdruck, dass die Divisormethode mit Standardrundung (Sainte-Laguë / Schepers) bei wiederholter Anwendung praktisch unverzerrte Sirzzahlen liefert und jeder Partei im Durchschnitt das zuteilt, was ihr von ihren Idealansprüchen her zusteht. Siehe Karsten Schuster / Friedrich Pukelsheim / Mathias Drton / Norman R. Draper, Seat biases of apportionment methods for proportional representation, in: Electoral Studies, 22. Jg. (2002), S. 651 - 676, S. 657.

29 Vgl. Michel Balinski / Friedrich Pukelsheim, Die Mathematik der doppelten Gerechtigkeit, in: Spektrum der Wissenschaft, April 2007, S. 76-80, S. 79.

30 Randnummern 66 und 71 im Urteil vom 6. Juli 1999 (VerfGH 14/98 und 15/98): www.justiz. nrw.de/nrwe/ovgs/vgh_nrwj/1999/VerfGH_14_98_VerfGH_15_98urteil19990706.html.

31 Geschäftsordnung des Landtags Nordrhein-Westfalen, Stand 15. März 2006. Siehe auch Matthias Hauser / Tobias Jaag, Zulässigkeir direkter Quoren bei kantonalen Parlamentswahlen, insbesondere bei den Grossratswahlen im Kanton Aargau, in: Schweizerisches Zentralblatt für Staats- und Verwal cungsrecht, 109. Jg. (2008) H. 2, S. 65-92. 
zahlenmäßig leichter nachzuvollziehen, um das Landeswahlgeserz vermittelnd einzuordnen zwischen dem Kommunalwahlgesetz einerseits und dem Bundeswahlgeserz andererseits.

\section{Erhöhungsstrategien sichern die Erfolgswertgleichheit der Wählerstimmen}

Wahlsysteme unterliegen vielfältigen Anforderungen. Sie sollen zu einer dem Grundsatz der Wahlgleichheit genügenden Verteilung der Sitze führen (representativeness), aber auch im so zusammengesetzten Parlament eine zielführende Regierungsbildung ermöglichen (governability). Bei Systemen, die den Grundsätzen einer mit der Personenwahl verbundenen Verhältniswahl folgen, können nicht immer alle Anforderungen mit einer festen, vorgegebenen Parlamentsgröße makellos erfüllt werden.

Hinsichtlich der Parlamentsgröße sind die jetzigen in Deutschland verwendeten Wahlsysteme durchaus flexibel. Beim Bundestag kann die Sitzzahl durch Überhangmandate anwachsen. Bei vielen Landtagen kommen beim Auftreten von Überhangmandaten zusärzlich Ausgleichsmandate hinzu. Daher wird hier dafür plädiert, die Variabilität der Parlamentsgröße innerhalb gewisser Grenzen konsequent zu Ende zu denken und die so gewonnene Bewegungsfreiheit zu nurzen, um den Wahlgrundsätzen (noch) besser gerecht zu werden.

Beim Anfall von Überhangmandaten bietet sich an, die Parlamentsgröße so lange zu erhöhen, bis keine Überhangmandate mehr übrig bleiben und alle Sitze von der Verhältnisrechnung getragen werden. Bei diesem Vorgehen erscheinen Überhangmandate nur als vorübergehende Rechengrößen; die auf dem Weg zur endgültigen Sitzzuteilung erzeugten weiteren Mandate können als Ausgleichsmandate interpretiert werden. Die im Ergebnis sich einstellende Sitzzuteilung wird aber allein aus der Verhältnisrechnung hergeleitet, Überhangmandate und Ausgleichsmandate haben im Endergebnis keinen Platz mehr.

Auch für die Auflösung von Gleichständen kann eine Vergrößerung des Parlaments empfohlen werden. Der derzeit in den meisten Gesetzen vorgesehene Losentscheid ist eher ein Relikr aus dem Mehrheitswahlsystem, bei dem jeweils nur eine einzige Person das Amt bekleiden kann. Losentscheide in Verhältniswahlsystemen führen dazu, dass eine Wählergruppe vom Losglück profitiert und eine andere das Nachsehen hat. Im Gegensatz dazu führt eine Erhöhung der Sitzzahl dazu, dass alle Wähler gleich (gut) behandelt werden.

Es bleibt noch der Fall, dass eine absolute Stimmenmehrheit immer auch zu einer absoluten Sitzmehrheit führen sollte. Für Parlamentswahlen in der Bundesrepublik gibt es kein praktisches Beispiel, in dem ein gegenteiliger Effekt aufgetreten wäre. Theoretisch kann man aber solche Stimmenkonstellationen konstruieren. Die Aufnahme von Mehrheitsklauseln in Wahlgesetze dürfte daher weniger von der Absicht des Gesetzgebers getragen sein, sich gegen unwirkliche theoretische Sonderfälle abzuschirmen. Eher dürfte im Vordergrund stehen, das Wählervotum sehr ernst zu nehmen und das funktionale Ziel zu erreichen, dass in einer parlamentarischen Demokratie die Regierungsbildung mit der Parlamentsmehrheit einhergeht. Auch hier bewährt sich die Erhöhungsstrategie.

Die Diskussion anhand der aus Nordrhein-Westfalen vorliegenden Daten zeigt, dass in aller Regel über die ausgangs vorgegebene Parlamentsgröße hinaus nur wenige Sizze hinzut kommen. Im Falle von Überhang- und Ausgleichsmandaten werden sogar weniger geschaffen als durch die jetzigen gesetzlichen Regelungen. Vor allem aber verwirklichr die schiließlich sich einstellende Sitzzuteilung aufgrund des systematischen Zugangs in hervorragender Weise den zentralen Grundsatz der Erfolgswertgleichheit der Wählerstimmen. 